\title{
Desenvolvimento de sorvete de chocolate utilizando fibra de casca de laranja como substituto de gordura
}

\author{
Development of chocolate ice cream using orange peel fiber as fat replacer \\ Camila Comas e Boff ${ }^{I}$ Tainara de Moraes Crizel ${ }^{I}$ Rubilene Ramos de Araujo \\ Alessandro de Oliveira Rios ${ }^{\mathrm{I}}$ Simone Hickmann Flôres ${ }^{\mathrm{I} *}$
}

\section{RESUMO}

A procura por alimentos mais saudáveis por parte dos consumidores tem impulsionado as indústrias de alimentos a desenvolverem alimentos que contenham menor teor de gordura, contudo sem alterar as características sensoriais e nutricionais dos produtos. A indústria de sorvetes é uma das que mais utiliza gordura em suas formulações, contendo, em média, 10\% desse ingrediente. Uma alternativa para a substituição de gorduras é a fibra de frutas cítricas, que vem sendo utilizada com sucesso, reduzindo o teor de gordura na indústria de embutidos, por exemplo. Nesse sentido, o objetivo deste trabalho foi avaliar as características sensoriais e físico-químicas de sorvetes elaborados com fibra de casca de laranja amarga comercial como substituto da gordura. Foram utilizadas duas formulações com concentrações diferentes de fibra de laranja (F1 com 0,74\% e F2 com 1,10\% de fibra) e uma formulação controle (com gordura). Para as duas formulações, foram encontrados valores condizentes com a literatura para a composição centesimal do sorvete. Em relação ao valor energético dos sorvetes, foi observada uma redução de mais de $25 \%$, indicando que o produto pode ter alegação de light. Para o teor de fibra dos sorvetes, os valores observados foram de F1 1,4\% e F2, 2,0\%, podendo considerar o sorvete da formulação F2 como fonte de fibras. Os atributos de cor, odor e textura não apresentaram diferenças entre as amostras. Já sabor, sabor residual e aceitação global obtiveram diferenças entre as amostras com fibra e a controle. Os sorvetes elaborados com fibra de casca de laranja obtiveram índices de aceitação de $78 \%$.

Palavras-chave: sorvete, fibra de laranja, análise sensorial, análises físico-químicas.

\section{ABSTRACT}

The demand for healthier foods by consumers has driven the food industry to develop foods that contain less fat, however without altering the sensory characteristics and nutritional products. The ice cream industry is one that uses more fat in their formulations, containing on average $10 \%$ of this ingredient. An alternative to the replacement of fat is the fiber of citrus fruits, which has been used successfully by reducing the fat content in the meat industry, for example. Thus, the objective of this study was to evaluate the sensory characteristics and physical-chemical ice cream made with fiber commercial bitter orange peel as a substitute for fat. Two formulations with different concentrations of orange fiber (F1 and F2 with $0.74 \%$ to $1.10 \%$ fiber) and a control formulation (with fat) were used. The two formulations were found consistent with the literature values for the chemical composition of ice cream. Regarding the energy value of ice cream was observed reduction of more than $25 \%$, indicating that the product may claim "light". For the fiber content of the cream, the values were $1.4 \%$ of F1 and F2, 2.0\%, can consider ice cream formulation F2 as a source of fiber. The attributes of color, odor and texture didn't differ between samples. Already flavor, after taste and overall acceptability differences between samples obtained with fiber and control. Ice cream prepared with fiber obtained orange peel acceptance rates of $78 \%$

Key words: ice cream, orange fiber, sensory evaluation, physicochemical.

\section{INTRODUÇÃO}

Os consumidores estão cada vez mais conscientes da relação entre alimentação e saúde. Buscando melhorar sua qualidade de vida, exigem alimentos mais saudáveis, que possuam ingredientes naturais seguros e que promovam benefício à saúde (SIRÓ et al., 2008). De acordo com RODRÍGUEZ et al. (2006), o consumo de fibras alimentares possui papel importante em muitos processos fisiológicos e na prevenção de algumas doenças. As fibras alimentares vêm sendo utilizadas como ingredientes no desenvolvimento de muitos produtos, não apenas

'Departamento de Ciência dos Alimentos, Universidade Federal do Rio Grande do Sul (UFRGS), 91501-970, Porto Alegre, RS, Brasil. E-mail: simone.flores@ufrgs.br.*Autor para correspondência. 
por suas propriedades nutricionais, mas também como potencial substituto de gordura (MENDOZA et al., 2001; THEBAUDIN et al., 1997).

A fibra dietética é constituinte significativo de muitos frutos, entre eles a laranja (GRIGELMOMIGUEL \& MARTÍN-BELLOSO, 1999). A utilização de subprodutos da indústria de sucos cítricos é muito interessante do ponto de vista nutricional, pois contêm várias substâncias como fibras, pectina, compostos bioativos e, do ponto de vista econômico, reduz a quantidade de resíduos da indústria (FERNÁNDEZ-LÓPEZ et al., 2009).

Segundo a Associação Nacional dos Exportadores de Sucos Cítricos (CITRUSBR, 2010), o Brasil possui produção equivalente a $25 \%$ da mundial, sendo o maior produtor de laranja do mundo, seguido pelos Estados Unidos. A Associação informa ainda que $70 \%$ da produção brasileira e dos Estados Unidos é destinada exclusivamente à produção de suco, gerando grande quantidade de subprodutos que podem ser utilizados como fonte para obtenção da fibra de laranja.

Considerando a fibra de laranja um subproduto da indústria de sucos pouco explorado como substituto de gordura e a crescente demanda de produtos mais saudáveis, como sorvetes com reduzido teor de gordura, este trabalho visou à utilização de fibras de laranja comercial na substituição de gordura em sorvete de chocolate, analisando suas características físicas, químicas e sensoriais.

\section{MATERIAL E MÉTODOS}

\section{Material}

A fibra de casca de laranja amarga foi adquirida da empresa Tango Alimentos, localizada na cidade de Curitiba, Paraná. Os demais ingredientes (leite desnatado UHT, leite em pó desnatado, creme de leite fresco ( $35 \%$ de gordura), açúcar) foram adquiridos no mercado local. A glicose, Glucosoft (dextrose), DPO super 100 (estabilizante) e o saborizante de chocolate (cacau em pó com $20 \%$ de manteiga de cacau) foram adquiridos na empresa Vidal Distribuidora de Máquinas LTDA.

Produção do sorvete

Para a produção do sorvete, foi utilizada uma planta de processamento descontínuo, situada na empresa Vidal Máquinas e Produtos para Sorvetes, na cidade de Porto Alegre. Foram elaboradas três formulações diferentes, sendo uma controle (C) com adição de gordura (5\%) e duas com diferentes concentrações de fibra de laranja (F1 e F2, com $0,74 \%$ e $1,10 \%$ de fibra, respectivamente). As formulações dos sorvetes (controle, F1 e F2) foram obtidas com base em testes preliminares, realizados em formulação fornecida pela empresa onde o sorvete foi processado (Tabela 1). Nas formulações F1 e F2, a fibra de laranja foi utilizada como substituto de gordura, sendo a única variação entre elas a quantidade de fibras adicionada.

Análises físico-químicas

Composição centesimal

O teor de umidade, cinzas, proteína (Nx6,38), lipídeos foi determinado segundo método da AOAC (1990). A determinação de fibra alimentar total foi realizada pelo método da AOAC (1990), no 991.43. Os carboidratos totais foram determinados por diferença. As análises foram realizadas em triplicata.

Valor energético total (VET)

Para o valor energético total das formulações (C, F1 e F2), foram utilizados os fatores de conversão $4 \mathrm{kcal} \mathrm{g}^{-1}$ para carboidratos e proteínas e 9 kcal g ${ }^{-1}$ para lipídeos, utilizando os valores em base úmida (BRASIL, 1998b).

\section{Overrun}

O overrun é utilizado para medir o aumento do volume do sorvete após o congelamento do mix. A incorporação de ar pode ser determinada de acordo com a equação (SEGALL \& GOFF, 2002):

$$
\text { Overrun }=\frac{\text { volume do sorvete } \mathbf{I} \text { volume do }{ }^{*} \text { mix" }}{\text { volume do }{ }^{*} \text { mix" }} \times 100
$$

\section{Taxa de derretimento}

A taxa de derretimento foi medida de acordo com OHMES et al. (1998), com algumas adaptações. As amostras utilizadas nos testes foram armazenadas a $-18^{\circ} \mathrm{C}$ durante 24 horas. Primeiramente, as amostras $(70 \mathrm{~g})$ foram colocadas em câmara de incubação, sem corrente de ar, à temperatura de $25^{\circ} \mathrm{C} \pm 1^{\circ} \mathrm{C}$, no topo de uma tela de arame, com malhas de $1 \mathrm{~cm}^{2}$, localizada sobre um Becker previamente tarado. Depois de 30 minutos, o Becker com amostra foi substituído e o sorvete derretido foi pesado. Tal processo foi repetido em intervalos de 10 minutos durante 70 minutos. $\mathrm{O}$ experimento foi realizado em triplicata para todas as amostras. Os resultados foram analisados a partir do gráfico do tempo versus a porcentagem de sorvete derretido. A regressão linear permitiu obter equações para o derretimento dos sorvetes e, a partir destas, obter a taxa de derretimento dos sorvetes. 
Tabela 1 - Composição das formulações de sorvete de chocolate da formulação controle - C (com gordura) F1 (com 0,74\% de fibra de casca de laranja) e F2 (com $1,10 \%$ de fibra de casca de laranja).

\begin{tabular}{|c|c|c|c|c|c|c|}
\hline Ingredientes & $\mathrm{C}(\mathrm{kg})$ & $\mathrm{C} \%$ & F1 (kg) & $\mathrm{F} 1 \%$ & F2 (kg) & $\mathrm{F} 2 \%$ \\
\hline Leite desnatado & - & - & 1 & 74,13 & 1 & 73,53 \\
\hline Leite integral & 0,876 & 64,41 & - & - & - & - \\
\hline Leite em pó desnatado & - & - & 0,010 & 0,74 & 0,010 & 0,74 \\
\hline Leite em pó integral & 0,068 & 5,00 & - & - & - & - \\
\hline Fibra de laranja & - & - & 0,010 & 0,74 & 0,015 & 1,10 \\
\hline Creme de leite fresco & 0,078 & 5,74 & - & - & - & - \\
\hline Açúcar & 0,150 & 11,03 & 0,135 & 10,00 & 0,135 & 10,00 \\
\hline Glicose & 0,057 & 4,19 & 0,015 & 1,11 & 0,015 & 1,20 \\
\hline Glucosoft & 0,022 & 1,62 & 0,015 & 1,11 & 0,015 & 1,10 \\
\hline DPO super 100 & 0,043 & 3,16 & 0,100 & 7,41 & 0,100 & 7,35 \\
\hline Saborizante de chocolate & 0,066 & 4,85 & 0,064 & 4,76 & 0,064 & 4,70 \\
\hline Total $(\mathrm{kg})$ & 1,360 & 100 & 1,349 & 100 & 1,360 & 100 \\
\hline
\end{tabular}

C: formulação controle; F1: formulação com $0,74 \%$ de fibra de laranja; F2: formulação com $1,10 \%$ de fibra de laranja.

Análise sensorial

A aceitação sensorial dos sorvetes foi realizada por 50 provadores não treinados, com idades entre 18 a 67 anos. Os testes foram realizados no Instituto de Ciência e Tecnologia de Alimentos da Universidade Federal do Rio Grande do Sul. As amostras foram servidas em copos descartáveis de $50 \mathrm{~mL}$, com cerca de $30 \mathrm{~g}$ de cada amostra, codificados com números de três dígitos aleatórios. Utilizouse escala hedônica de nove pontos, com os pontos extremos 1 - "Desgostei Muitíssimo" e 9 - "Gostei Muitíssimo" (DUTCOSKY, 1996). Foram avaliados os atributos cor, odor, sabor, sabor residual, textura e aceitação global. Para cálculo do percentual de aceitação de cada atributo, utilizou-se a seguinte equação:

$\% \quad$ Aceitação $=\frac{\text { Média de Aceitação }}{9} \times 100 \quad \%$ (DUTCOSKY, 1996).

Foi realizada a análise de intenção de compra de cada um dos sorvetes (controle, F1 e F2) e, para isso, os julgadores foram questionados se comprariam a amostra que estava sendo analisada. A porcentagem de julgadores que respondeu "sim" foi calculada com base no número total de julgadores que realizaram o teste.

Análise estatística

Todos os resultados obtidos a partir das análises (físico-químicas e sensorial) foram submetidos às análises estatísticas de ANOVA, teste de comparação de médias de Tukey ao nível de 5\% de significância, através do programa Statistica 10.0 (CALADO \& MONTOGMERY, 2003).

\section{RESULTADOS E DISCUSSÃO}

Análises físico-químicas

$\mathrm{O}$ teor de umidade encontrado nas diferentes formulações dos sorvetes variou de $63 \%$ a $70 \%$ (Tabela 2). O sorvete controle apresentou menor valor de umidade do que o sorvete adicionado de fibra. Segundo PINHEIRO \& PENNA (2004), na formulação do sorvete, quanto maior a quantidade de gordura, menor a quantidade de água e isso contribui para retardar o derretimento do produto.

Os sorvetes elaborados com fibra de laranja apresentaram um menor valor no teor de lipídeos, diferindo significativamente $(\mathrm{P} \leq 0,05)$ quando comparados ao sorvete padrão (com gordura). Houve uma redução de $70,4 \%$ e $71,4 \%$ no teor de gordura do sorvete adicionado de $0,74 \%$ e $1,10 \%$ de fibra, respectivamente, em relação ao sorvete controle. Porém, a diferença no teor de gordura dos sorvetes adicionados de diferentes concentrações de fibra de laranja não foi significativa.

Não houve diferença significativa $(\mathrm{P}>0,05)$ em relação ao teor de proteína das diferentes formulações de sorvete. SILVA JUNIOR \& LANNES (2011) avaliaram as propriedades químicas, físicas e mecânicas de sorvetes de chocolate, produzidos com misturas de açúcar e tipos diferentes de gordura, e observaram que o conteúdo de proteína nas diferentes formulações não variou.

O comportamento do sorvete durante o derretimento variou entre as três diferentes 
Tabela 2 - Análises físico-químicas dos Sorvetes F1, F2 e Controle.

\begin{tabular}{|c|c|c|c|}
\hline \multirow{2}{*}{ Análises } & \multirow[b]{2}{*}{$\mathrm{F} 1$} & \multirow[b]{2}{*}{$\mathrm{F} 2$} & \multirow[b]{2}{*}{ Controle } \\
\hline & & & \\
\hline Umidade (\%) & $70,03 \pm 1,28^{\mathrm{a}}$ & $69,97 \pm 0,04^{\mathrm{a}}$ & $63,03 \pm 0,45^{\mathrm{b}}$ \\
\hline Proteína (\%) b.s & $16,32 \pm 0,09^{\mathrm{a}}$ & $15,38 \pm 0,23^{\mathrm{a}}$ & $12,87 \pm 0,07^{\mathrm{a}}$ \\
\hline Lipídeos (\%) b.s & $5,47 \pm 0,04^{\mathrm{a}}$ & $5,29 \pm 0,02^{\mathrm{a}}$ & $18,53 \pm 0,01^{\mathrm{b}}$ \\
\hline Cinzas (\%) b.s & $4,17 \pm 0,01^{\mathrm{a}}$ & $4,09 \pm 0,02^{\mathrm{a}}$ & $3,41 \pm 0,03^{\mathrm{a}}$ \\
\hline Carboidratos (\%) b.s & $69,94 \pm 1,35^{\mathrm{a}}$ & $69,20 \pm 0,20^{\mathrm{a}}$ & $65,21 \pm 0,42^{\mathrm{a}}$ \\
\hline Fibra Alimentar (\%) b.s & 0,70 & 1,03 & - \\
\hline $\operatorname{VET}\left(\left(\mathrm{kcal} 100 \mathrm{~g}^{-1}\right)\right.$ & 121,9645 & 123,7022 & 177,6174 \\
\hline Taxa de derretimento $\left(\% \min ^{-1}\right)$ & $2,43 \pm 0,09^{\mathrm{ab}}$ & $2,24 \pm 0,08^{b}$ & $2,48 \pm 0,08^{\mathrm{a}}$ \\
\hline Overrun $(\%)$ & $29 \%$ & $25 \%$ & $46 \%$ \\
\hline
\end{tabular}

* Resultados são médias de três determinações \pm desvio padrão. Letras diferentes na mesma linha diferem significativamente pelo teste de Tukey $(\mathrm{P} \subseteq 0,05)$.

C: formulação controle; F1: formulação com $0,74 \%$ de fibra de laranja; F2: formulação com 1,10\% de fibra de laranja; b.s: base seca; VET: Valor Energético Total.

formulações. O sorvete que apresentou a menor taxa de derretimento foi a formulação F2 2,24\% $\min ^{-1}$, porém não apresentou diferença significativa quando comparado à formulação $\mathrm{F} 12,43 \% \mathrm{~min}^{-1}$. O sorvete que obteve a maior taxa de derretimento foi o controle, com $2,48 \% \mathrm{~min}^{-1}$, apresentando diferença significativa da formulação F2, indicando assim que a adição de 1,10\% de fibra, em substituição à gordura, auxiliou na diminuição da velocidade de derretimento do sorvete.

Estudo realizado por PRINDIVILLE et al. (2000) mostrou que a adição de substitutos de gordura a base de proteína em sorvetes de chocolate aumentou a taxa de derretimento dos sorvetes em relação à amostra controle ( com gordura de leite), resultados que foram contrários aos obtidos neste estudo.

De acordo com GOFF (2002), o volume de ar incorporado pode ser de mais de $50 \%$ a um mínimo de $10 \%$ a $15 \%$. Os três sorvetes obtidos neste estudo apresentaram porcentagens maiores que os mínimos, mas não chegaram ao máximo, apresentados por Goff.

DERVISOGLU \& YAZICI (2006), usando também fibras provenientes de frutas cítricas, obtiveram valores para o overrun próximos aos observados utilizando fibra de laranja como substituto de gordura. Os autores encontraram valores entre $39 \%$ e $29 \%$ usando somente a fibra e, entre $39 \%$ e $22 \%$, utilizando uma mistura de emulsificantes e a fibra. Foi observado que a adição de fibras reduz o overrun obtido nos sorvetes, já que estas aumentam a viscosidade do "mix", reduzindo a incorporação de ar, o que condiz com os resultados encontrados neste estudo. YILSAY et al. (2006) também observaram uma redução no valor do overrun de sorvetes de baunilha, quando adicionaram proteína de soro de leite em substituição à gordura.

A fibra obtida nos dois sorvetes variou de acordo com a quantidade adicionada. $\mathrm{Na}$ formulação $\mathrm{F}$ 1, foram obtidos $0,21 \mathrm{~g}$ de fibras por $100 \mathrm{~g}$ de sorvete e, na formulação $\mathrm{F} 2$, foram obtidos $0,31 \mathrm{~g}$ de fibras por $100 \mathrm{~g}$ de sorvete. De acordo com o Ministério da Saúde, Portaria no 27, de 13 de janeiro de 1998 (BRASIL,1998a), um alimento fonte de fibras deve conter mais do que $1,5 \mathrm{~g}$ de fibra por $100 \mathrm{~g}$, sendo assim, nenhum dos sorvetes pode ser apresentado com essa alegação.

Os sorvetes com adição de fibra (F1 e F2) e o controle, apresentaram densidade 17 aparente de 775,800 e $685 \mathrm{~g} \mathrm{~L}^{-1}$, respectivamente, atendendo ao requisito específico de 18 densidade aparente mínima de $475 \mathrm{~g}$ litro $^{-1}$, estabelecido pela ANVISA em sua Resolução RDC 19 no 266, de 22 de setembro de 2005 (BRASIL, 2005).

SALES et al. (2008) desenvolveram um sorvete de creme enriquecido de granola e frutooligossacarídeos (FOS), que apresentou $5,45 \mathrm{~g}$ de fibra por $100 \mathrm{~g}$ de sorvete, valor superior ao encontrado para F2. Isso demonstra que a concentração de fibra de laranja adicionada ao sorvete deste estudo não foi suficiente para elevar o valor nutricional do produto para que este pudesse ser classificado como fonte de fibras.

As formulações F1 e F2, contendo a fibra, apresentaram redução no valor energético total em relação à amostra controle. A amostra F1 apresentou uma redução de 31,33\% (redução de 55,64kcal $100 \mathrm{~g}^{-1}$ ) e a amostra F2 apresentou redução de $30,35 \%$

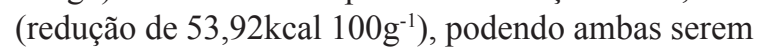


consideradas como light pela legislação brasileira (BRASIL, 1998a).

Análise sensorial

Verificou-se que, entre as características analisadas de cor, odor e textura, não houve diferenças significativas entre as três amostras de sorvetes (Tabela 3 ), sendo que todas as amostras receberam notas acima de 7 (equivalente a gostei moderadamente) para esses atributos. Entretanto, a amostra controle obteve as maiores médias para os atributos de sabor, sabor residual e aceitação global, diferindo significativamente dos demais sorvetes testados.

Em relação aos atributos de sabor e sabor residual, as amostras F1 e F2 não obtiveram diferença significativa entre si, indicando que as diferentes concentrações de fibra adicionadas aos sorvetes não foram determinantes na avaliação desses atributos. De acordo com DERVISOGLU \& YAZICI (2006), que utilizaram $0,8 \%$ e $1,2 \%$ de fibra proveniente de frutas cítricas na produção de sorvetes, a adição da fibra reduziu as notas atribuídas pelos julgadores para o sabor, diferindo significativamente da amostra controle (sem adição de fibra). Porém, quando houve a mistura da fibra com emulsificantes, as notas atribuídas foram maiores ou próximas as da amostra controle.

As notas baixas, que foram dadas pelos julgadores para os atributos de sabor e sabor residual dos sorvetes adicionados de fibra de laranja, podem ser justificadas pela falta de tratamento prévio no subproduto utilizado para elaboração da fibra, com o objetivo de retirar os compostos responsáveis pelo sabor amargo, tais como óleos essenciais e naringina, presentes na casca e bagaço de laranja.

$\mathrm{Na}$ aceitação global, apesar de ter havido diferenças significativas entre as amostras F1 e C e entre F2 e C, as médias obtidas para os sorvetes com substituição de gordura por fibra de laranja ficaram próximas da nota 7 (equivalente a gostei moderadamente), demostrando um percentual de aceitação de 78\%. Segundo DUTCOSKY (1996), percentual de aceitação acima de $70 \%$ indica que o produto tem um potencial mercadológico.

Em relação à intenção de compra dos sorvetes analisados pelos provadores, o sorvete C (controle) obteve a maior porcentagem de intenção de compra com 96\%, seguido da amostra F1 com 74\% e da F2 com 64\% de intenção, indicando que, apesar das menores médias de aceitação que foram atribuídas ao sabor dos sorvetes com fibras, a amostra F1 conquistou uma parcela dos julgadores, o que poderia levar a uma boa comercialização do produto. A partir de comentários realizados pelos provadores durante o teste sensorial, foi possível verificar que, se uma alegação funcional fosse atribuída ao produto, este teria uma maior intenção de compra por parte dos consumidores.

\section{CONCLUSÃO}

A fibra de casca de laranja amarga utilizada como substituto de gordura em sorvetes se apresentou como uma alternativa na elaboração de sorvete light, obtendo aceitação global de $78 \%$ e mantendo a maioria de suas características químicas.

A fibra manteve as características físicas e químicas do sorvete de chocolate e reduziu o valor energético total, podendo ser classificado como light, já que a presença de fibra reduziu mais do que $25 \%$ o valor energético.

Para outros estudos, seria interessante realizar um tratamento prévio na fibra, reduzindo assim o sabor de laranja e retirando os compostos responsáveis pelo amargor da fibra, possibilitando aumento das notas dos provadores em relação a sabor e sabor residual e, consequentemente, aumentando as notas da aceitação global.

Tabela 3 - Aceitação sensorial dos atributos das formulações dos sorvetes (controle, F1 e F2).

\begin{tabular}{lccc}
\hline & & & \\
Atributos & F1 & F2 & C \\
\hline Cor & $7,58 \pm 1,25^{\mathrm{a}}$ & $7,88 \pm 1,30^{\mathrm{a}}$ & $8,12 \pm 1,04^{\mathrm{a}}$ \\
Odor & $7,36 \pm 1,24^{\mathrm{a}}$ & $7,28 \pm 1,40^{\mathrm{a}}$ & $7,36 \pm 1,35^{\mathrm{a}}$ \\
Sabor & $6,48 \pm 1,66^{\mathrm{b}}$ & $6,62 \pm 1,71^{\mathrm{b}}$ & $8,12 \pm 0,94^{\mathrm{a}}$ \\
Sabor Residual & $5,78 \pm 1,66^{\mathrm{b}}$ & $6,06 \pm 1,75^{\mathrm{b}}$ & $7,70 \pm 1,11^{\mathrm{a}}$ \\
Textura & $7,62 \pm 1,38^{\mathrm{a}}$ & $7,40 \pm 1,56^{\mathrm{a}}$ & $8,06 \pm 1,28^{\mathrm{a}}$ \\
Aceitação Global & $6,86 \pm 1,30^{\mathrm{b}}$ & $6,96 \pm 1,56^{\mathrm{b}}$ & $8,01 \pm 0,68^{\mathrm{a}}$ \\
\hline
\end{tabular}

*Letras minúsculas iguais nas colunas não diferem estatisticamente pelo Teste de Tukey em nível de 5\% de significância. C: formulação controle; F1: formulação com $0,74 \%$ de fibra de laranja; F2: formulação com $1,10 \%$ de fibra de laranja. 


\section{COMITÊ DE ÉTICA}

Para participar da pesquisa, todos os provadores assinaram o Termo de Consentimento Livre e Esclarecido, aprovado pelo Comitê de Ética em Pesquisa da UFRGS (Protocolo no 21912).

\section{REFERÊNCIAS}

AOAC. Official methods of analysis. 15.ed. Arlington: Association of Official Analytical Chemist, 1990. V.2. 1298p.

BRASIL. Agência Nacional de Vigilancia Sanitária - ANVISA Portaria n.27 de 13 de janeiro de 1998a. Disponível em: <http:/ www.anvisa.gov.br/legis/portarias/27 98.htm>. Acesso em: 10 set. 2011.

BRASIL. Agencia Nacional de Vigilancia Sanitaria - ANVISA. Resolucao RDC n² 266, de 22 de setembro de 2005 Disponível em: <http://portal.anvisa.gov.br/wps/portal/anvisa/anvisa/home/ alimentos>. Acesso em: 13 fev. 2013

BRASIL. Agência Nacional de Vigilancia Sanitária - ANVISA Portaria n.41, de 14 de janeiro de 1998b. Aprova regulamento técnico referente a rotulagem nutricional de alimentos embalados. Diário Oficial da União. Brasília, 21 de jan. 1998. Disponível em: $<\mathrm{http}$ ://www.anvisa.gov.br/legis/portarias/41 98.htm>. Acesso em: 10 set. 2011

CALADO, V.; MONTGOMERY, D. Planejamento de experimentos usando o Statistica. Rio de Janeiro: E-papers Servicos Editorais, 2003. 260p.

CITRUSBR - Associação Nacional dos Exportadores de Sucos Cítricos. Retrato da citricultura (2010). Disponível em: $<$ http:// www.citrusbr.com/exportadores-citricos/brasil/retrato-dacitricultura-256277-1.asp>. Acesso em: 12 set. 2011.

DERVISOGLU, M; YAZICI, F. Note. Effect of citrus fibre on the physical, chemical and sensory of ice cream. Food Science and Technologi International, v.12, p.159-164, 2006. Disponível em: $<$ http://fst.sagepub.com/content/12/2/159.abstract $>$. Acesso em: 20 mar. 2012. doi: 10.1177/1082013206064005.

DUTCOSKY, S.D. Análise sensorial de alimentos. Curitiba: Champagnat, 1996. 123p.

FERNÁNDEZ-LÓPEZ, J. et al. Storage stability of a high dietary fibre powder from orange by-products. International Journal of Food Science \& Technology, v.44, p.748-756, 2009. Disponível em: <http://onlinelibrary.wiley.com/doi/10.1111/j.13652621.2008.01892.x/abstract>. Acesso em: 28 jan. 2012. doi:10.111 $1 / \mathrm{j} .1365-2621.2008 .01892$.

GRIGELMO-MIGUEL, N.; MARTÍN-BELLOSO, O. Comparison of dietary fibre from by-products of processing fruits and greens and from cereals. Lebensmittel Wissenschaft Und-Technologie, v.32, p.503-508, 1999. Disponível em: $<$ http://www.sciencedirect. com/science/article/pii/S002364389990587X>. Acesso em: 04 abr. 2012. doi: 10.1006/fstl.1999.0587.

GOFF, H.D. Formation and stabilisation of structure in ice cream and related products. Current Opinion in Colloid and Interface Science, v.7, p.432-437, 2002. Disponível em: <http://www. sciencedirect.com/science/article/pii/S1359029402000766> Acesso em: 05 mar. 2012. doi: 10.1016/s1359-0294(02)00076-6.
MENDOZA, E. et al. Inulin as fat substitute in low fat, dry fermented sausages. Meat Science, v.57, p.387-393, 2001. Disponível em: $<$ http:/www.ncbi.nlm.nih.gov/pubmed/22061711>. Acesso em: 06 mar. 2012. doi: 10.1016/S0309-1740(00)00116-9.

OHMES, R.L. et al. Sensory and physical properties of ice creams containing milk fat or fat replacers. Journal of Dairy Science, v.81, p.1222-1228, 1998. Disponível em: <http://www.ncbi. nlm.nih.gov/pubmed/9621223>. Acesso em: 01 fev. 2012. doi: 10.3168/jds.S0022-0302(98)75682-6.

PINHEIRO, M.V.S.; PENNA, A.L.B. Substitutos de gordura: tipos e aplicações em produtos lácteos. Alimentos e Nutrição, v.15, n.2, p.175-186, 2004. Disponível em:<http://serv-bib.fcfar.unesp.br/ seer/index.php/alimentos/article/viewFile/72/87>. Acesso em: 25 jan. 2012

PRINDIVILLE, E.A. et al. Effect of milk fat, cocoa butter, and whey protein fat replacers on the sensory properties of lowfat and nonfat chocolate ice cream. Journal of Dairy Science, v.83, p.2216-2223, 2000. Disponível em: <http://www.ncbi.nlm.nih. gov/pubmed/11049061>. Acesso em: 01 fev. 2012. doi:10.3168/ jds.S0022-0302(00)75105-8.

RODRÍGUEZ, R. et al. Dietary fibre from vegetable products as source of functional ingredients. In: Trends in Food Science \& Technology, v.17, cap.1,p.3-15,2006. Disponível em: $<$ http://www. sciencedirect.com/science/article/pii/S092422440500244X $>$. Acesso em: 06 mar. 2012. doi: 10.1016/j.foodchem.2010.06.077.

SALES, R.L et al. Mapa de preferência de sorvetes ricos em fibra. Ciência e Tecnologia de Alimentos, v.28, p.2731, 2008. Disponível em: <http://www.scielo.br/pdf/cta/ v28s0/05.pdf>. Acesso em: 20 mar. 2012. doi: 10.1590/S010120612008000500005

SEGALL, K.I; GOFF, H.D. A modified ice cream processing routine that promotes fat destabilization in the absence of added emulsifier. International Dairy Journal, v.12, p.1013-1018, 2002. Disponível em: <http://www.sciencedirect.com/science/ article/pii/S0958694602001176>. Acesso em: 01 fev. 2012. doi: 10.1016/s0958-6946(02)00117-6.

SILVA JUNIOR, E.; LANNES, S.C.S. Effect of different sweetener blends and fat types on ice cream properties. Revista Ciência e Tecnologia de Alimentos, v.31, p.217-220, 2011. Disponível em: <http://www.scielo.br/scielo.php?script=sci_artte xt\&pid=S0101-20612011000100033 > . Acesso em: 20 mar. 2012. doi: 10.1590/S0101-20612011000100033.

SIRÓ, I. et al. A funcional food. Product development, marketing and consumer accenptance - A review. Appetite, v.51, n.3, p.456-467, 2008. Disponível em: <http://www.ncbi.nlm.nih.gov/ pubmed/18582508>. Acesso em: 01 fev. 2012. doi: 10.1016/j. appet.2008.05.060.

THEBAUDIN, J.Y. et al. Dietary fibres: nutritional and technological interest. Trends in Food Science \& Technology, v.8, p.41-48, 1997. Disponível em: <http://www.sciencedirect. com/science/article/pii/S0924224497010078>. Acesso em: 03 fev. 2012. doi: 10.1016/s0924-2244(97)01007-8

YILSAY, T.O. et al. The effect of using a whey protein fat replacer on textural and sensory characteristics of low-fat vanilla ice cream. European Food Research and Technology, v.222, p.171-175, 2006. Disponível em: <http://link.springer.com/ article/10.1007\%2Fs00217-005-0018-x>. Acesso em: 10 abr. 2012. doi: $10.1007 / \mathrm{s} 00217-005-0018-\mathrm{x}$. 